[0212-7199 (2007) 24: 9; pp 439-441] ANALES DE MEDICINA INTERNA Copyright (C) 2007 ARAN EDICIONES, S.L.

AN. MED. INTERNA (Madrid) Vol. 24, N. ${ }^{\circ} 9$, pp. 439-441, 2007

\title{
Complicaciones neurológicas como manifestación inicial de endocarditis infecciosa
}

\author{
J. F. VARONA \\ Departamento de Medicina Interna. Hospital El Escorial. San Lorenzo de El Escorial. \\ Madrid
}

\author{
NEUROLOGICAL MANIFESTATIONS AS PRESENTATION OF \\ INFECTIOUS ENDOCARDITIS
}

\begin{abstract}
RESUMEN
Las complicaciones neurológicas de la endocarditis infecciosa (EI) son en ocasiones la primera manifestación clínica de la enfermedad y están asociadas con un importante incremento en su mortalidad. La principal forma de presentación es el ictus embólico, pero se han descrito otras muchas que abarcan desde la hemorragia cerebral hasta la excepcionalmente descrita meningitis aséptica acelular. Presentamos 3 casos ilustrativos de este amplio espectro clínico. El diagnóstico de ictus isquémico cerebral en el primer paciente y de hemorragia intraparenquimatosa en el segundo precedió en varios días a la documentación de la EI. En el tercer caso un cuadro de meningitis aguda acelular fue la inusual forma de debut de EI. Debido a que muy raramente la EI es la causa subyacente a un infarto o a una hemorragia cerebral, es fundamental tener un alto grado de alerta para reconocer precozmente estas complicaciones y así establecer el diagnóstico de EI, iniciando rápidamente el tratamiento antibiótico y planteando el recambio valvular y el momento adecuado del mismo, para disminuir el riesgo de deterioro neurológico y evitar el desarrollo de nuevas complicaciones que empeoren el pronóstico.
\end{abstract}

PALABRAS CLAVE: Endocarditis infecciosa. Complicaciones. Sistema nervioso. Meningitis aséptica. Isquemia cerebral.

\begin{abstract}
Neurologic complications of infective endocarditis (IE) are frequent. In many cases, they are the initial feature and considerably impair the prognosis of the disease. The most common neurologic manifestation is embolic stroke, but other many neurologic events have been described, ranged from cerebral hemorrhage due to rupture of mycotic aneurysm to the exceptional aseptic meningitis with acellular cerebrospinal fluid. We describe 3 cases that represent this wide spectrum of presentation. Ischemic stroke in the first patient and cerebral hemorrhage in the second were respectively documented several days before the diagnosis of $I E$. In the third case, acellular meningitis was the unusual clinical debut of aortic IE. Cerebral infarct or hemorrhage are exceptionally caused by an underlying IE, so a high level of suspicious is needed to recognize these complications. The best management to improve the prognosis has to be based on a rapid diagnosis and onset of antibiotic treatment, considering valve replacement in the adequate timing.
\end{abstract}

KEY WORDS: Endocarditis. Complications. Nervous system. Aseptic meningitis. Cerebral ischemia.

Varona JF. Complicaciones neurológicas como manifestación inicial de endocarditis infecciosa. An Med Interna (Madrid) 2007; $24: 439-441$.

\section{INTRODUCCIÓN}

Las complicaciones neurológicas son frecuentes en el curso de la endocarditis infecciosa (EI), apareciendo en el 20$40 \%$ de casos (1-4). Su reconocimiento es fundamental ya que con frecuencia constituyen la manifestación inicial de esta enfermedad.

Describimos a continuación los casos de 3 pacientes con EI que debutaron con un cuadro neurológico y que son representativos del amplio espectro clínico de presentación: desde el relativamente frecuente ictus embólico a la menos habitual hemorragia cerebral y a la excepcionalmente descrita meningitis aséptica acelular.

\section{CASOS APORTADOS}

Caso 1. Varón de 73 años recientemente intervenido de prostatectomía por hipertrofia prostática que presenta un cuadro de inestabilidad en la marcha y alteraciones visuales sin otra sintomatología. En el examen físico se encuentra afebril, orientado, sin signos meníngeos y destacando la presencia de una hemianopsia homónima izquierda. En la analítica no hay hallazgos relevantes. Se practica una tomografía axial computarizada (TAC) craneal que muestra una lesión isquémica en territorio frontera entre las arterias cerebrales media y posterior derechas. Es ingresado con el diagnóstico de ictus isquémico hemisférico derecho. A las 12 horas presenta fiebre sin focalidad clínica infecciosa aparente. Se practica una punción lumbar sin obtener anomalías en el análisis cito-bioquímico del líquido

Trabajo aceptado: 19 de abril de 2007

Correspondencia: J. F. Varona. Medicina Interna. Hospital de El Escorial. Ctra. M-600 de Guadarrama a San Lorenzo de El Escorial Km 6,255. 28200 San Lorenzo de El Escorial. E-mail: jfva_varona@yahoo.com 
cefalorraquideo (LCR), una ecografía abdominal que muestra una imagen sugerente de infarto esplénico y se extraen hemocultivos, en los que se aísla Enterococcus faecalis. Se realiza un ecocardiograma transesofágico que objetiva la existencia de una vegetación de $5 \mathrm{~mm}$ en la valva coronaria derecha de la válvula aórtica con insuficiencia aórtica severa, por lo que se establece el diagnóstico de endocarditis infecciosa enterocócica aórtica sobre válvula nativa complicada con fenómenos embólicos mayores (infarto cerebral e infarto esplénico), instaurándose terapia intravenosa con Ampicilina y Gentamicina, y a los 14 días, se practica recambio valvular aórtico con evolución postoperatoria satisfactoria.

Caso 2. Varón de 33 años, natural de Sudamérica, que ingresa por un cuadro de hemiparesia izquierda de 2 días de evolución. En el examen físico destaca la existencia de un soplo cardiaco sistodiastólico mitral y un déficit motor facio-braquio-crural izquierdo $3 / 5$. Se realizan estudios complementarios, con hallazgos analíticos anodinos y objetivándose en la TAC craneal un área de hemorragia cerebral a nivel del lóbulo temporal derecho. Se establece el diagnóstico de hemorragia cerebral de causa indeterminada en adulto joven y el paciente es ingresado para completar estudio etiológico. Se practica una arteriografía cerebral que no muestra evidencia de oclusión, malformaciones arterio-venosas cerebrales ni dilataciones aneurismáticas. A los pocos días del ingreso el paciente desarrolla fiebre sin focalidad clínica infecciosa, por lo que se realiza análisis del LCR, que no muestra hallazgos significativos, y se extraen hemocultivos, en los que se aísla Streptococcus bovis. Se practica un ecocardiograma que documenta la existencia de una vegetación de 8 × $6 \mathrm{~mm}$ a nivel de la válvula mitral que presentaba alteraciones degenerativas compatibles con una valvulopatía reumática mitral con doble lesión moderada. Se instaura terapia antibiótica parenteral guiada por antibiograma y el paciente presenta evolución clínica favorable. Los estudios de imagen cerebral practicados con posterioridad (días 12 y 30 desde el ingreso) muestran una imagen compatible con infarto cerebral subagudo temporal derecho con resolución de la hemorragia. El paciente es diagnosticado de EI mitral (sobre una valvulopatía reumática de base) complicada con un infarto hemorrágico cerebral y es sometido a recambio valvular pasadas 4 semanas.

Caso 3. Varón de 59 años sin antecedentes médicos relevantes que acudió a Urgencias por fiebre alta, malestar general y cefalea holocraneal de varios días de evolución, sin respuesta a terapia empírica ambulatoria con Amoxicilina-clavulánico. En el examen físico inicial el paciente estaba postrado, febril, con rigidez de nuca y signos meníngeos positivos, con auscultación cardiopulmonar anodina. La analítica no mostraba hallazgos reseñables. La TAC craneal con contraste intravenoso mostró una captación meníngea difusa y patológica y la punción lumbar dio salida a LCR turbio a hiperpresión (360 mm de agua), con hipoglucorraquia (49 mg/dl; glucemia: $113 \mathrm{mg} / \mathrm{dl})$, hiperproteinorraquia $(1,8 \mathrm{~g} / \mathrm{l})$ y ausencia de pleocitosis (0 células). La tinción Gram no mostró gérmenes y el cultivo de LCR fue estéril. Se inició antibioterapia empírica intravenosa (Ceftriaxona y Vancomicina) y fue ingresado con el diagnóstico de meningitis aguda aséptica de probable causa bacteriana decapitada. Los hemocultivos fueron repetidamente negativos. A los pocos días del ingreso el paciente persistía febril y comenzó con disnea, objetivándose la aparición de un soplo cardiaco aórtico, practicándose un ecocardiograma que documentó una gran vegetación de 16 x 12 mm en la valva sigmoidea anterior aórtica que condicionaba una insuficiencia aórtica moderada. Se asumió el diagnóstico de endocarditis infecciosa con 1 criterio de Duke mayor (ecocardiográfico) y solo 2 menores (fiebre y fenómenos inmunológicos en forma de afectación meníngea) dada la falta de documentación microbiológica por la instauración previa de antibioterapia empírica de amplio espectro. Con el juicio clínico de endocarditis infecciosa aórtica, sin filiación microbiológica, complicada con insuficiencia aórtica moderada y meningitis aguda aséptica acelular, el paciente pasó a la Unidad Coronaria, instaurándose terapia diurética y reforzándose la cobertura antibiótica con Ampicilina. A las 24 horas, tras una mejoría clínica inicial y a la espera de cirugía cardiaca urgente, el paciente entró en asistolia y parada cardio-respiratoria refractaria a maniobras prolongadas de resucitación cardiopulmonar avanzada.

\section{DISCUSIÓN}

Las complicaciones neurológicas de la endocarditis infecciosa (EI) son frecuentes habiéndose descrito su aparición hasta en el $20-40 \%$ de casos. Con frecuencia son la manifestación inicial de la enfermedad (1-5), presentándose antes de que llegue a establecerse el diagnóstico de EI e iniciarse el tratamiento. Este es el principal motivo de que su frecuencia y gravedad no haya disminuido en las últimas décadas, a pesar de la notable mejora en las técnicas diagnósticas y en los tratamientos antibióticos.

La embolización de fragmentos de las vegetaciones cardiacas a los vasos sanguíneos cerebrales es el principal mecanismo patogénico y su frecuencia se estima muy alta, ya sea de forma clínicamente silente (pequeños émbolos cerebrales sin expresividad clínica) o como un infarto cerebral sintomático Los principales factores de riesgo de embolización, y por tanto de complicaciones neurológicas, en la EI son el retraso en el inicio de la terapia antibiótica (un $76 \%$ de las complicaciones neurológicas ocurren antes de su instauración), la localización en cavidades izquierdas, el tamaño de la vegetación $>10 \mathrm{~mm}$ y la etiología por S. aureus y Streptococcus bovis $(1,6-8)$. La diseminación infecciosa a distancia y las lesiones mediadas inmunológicamente son otros mecanismos patogénicos reconocidos.

Las principales manifestaciones neurológicas de la EI son el ictus isquémico embólico (14-30\%), la hemorragia cerebral (3-5\%), la encefalopatía aguda (6\%), la meningitis (7\%), los abscesos cerebrales (2\%) y las crisis (1-4).

El infarto cerebral embólico (secundario a una embolia séptica o aséptica) es la presentación neurológica más común, siendo la manifestación clínica inicial en un 14\% de casos de EI. Es más frecuente en la EI de localización mitral y asocia una alta mortalidad próxima al 50\% $(3,9)$.

La hemorragia cerebral asociada a la EI es la complicación que mayor mortalidad asocia y puede ser subaracnoidea o intrapatrenquimatosa (generalmente de localización lobar). Se debe a tres mecanismos fundamentales: la vasculitis cerebral séptica (que provoca la ruptura del vaso tras el daño en su pared ocasionado por la impactación de un émbolo); la ruptura de aneurismas micóticos (en este caso la complicación neurológica suele ser tardía); y la transformación hemorrágica de un infarto cerebral (infarto hemorrágico), como ocurrió en el segundo caso que describimos. Esta última condición es habitual en los infartos por embolias sépticas y con frecuencia es asintomática, aunque cuando se asocia con el uso de anticoagulantes puede conllevar una importante deterioro neurológico (10).

La meningitis aguda asociada a la endocarditis bacteriana se debe por lo general a gérmenes virulentos, muy infrecuentemente es la manifestación inicial de la EI y presenta típicamente un perfil aséptico en el LCR con discreta pleocitosis mononuclear. En su mecanismo patogénico se implica el desarrollo de microabscesos cerebrales secundarios a embolización séptica $(1,2,11)$. La ausencia de pleocitosis hace excepcional la forma de presentación de la meningitis aguda con la que debutó la EI del tercer caso descrito. El mecanismo patogénico de este tipo de afectación meníngea (con recuento 
celular normal en el LCR) en la EI no es claro, pero está descrito excepcionalmente con gérmenes poco virulentos (como el Streptococci grupo B) (12), postulándose un origen inmunológico-inflamatorio o el desarrollo de múltiples microembolismos asépticos en las meninges (4).

La aparición de complicaciones neurológicas ensombrece dramáticamente el pronóstico de la EI, incrementando hasta 3.2 veces la mortalidad $(1,3)$. En este sentido es fundamental su reconocimiento precoz ya que el riesgo de embolismo cerebral disminuye a menos de un $5 \%$ después de 48 horas de controlada la infección (2).

El manejo de las complicaciones neurológicas de la EI es debatido, planteándose varios dilemas. La anticoagulación no se considera indicada en las embolias cerebrales asociadas a endocarditis de válvulas nativas ya que aumenta el riesgo de transformación hemorrágica sintomática $(2,13)$, mientras que sí se recomienda en la EI sobre prótesis o ante la existencia de otras fuentes embólicas como fibrilación auricular (3). Otro dilema lo constituye la indicación de cirugía valvular y el momento de la misma. Clásicamente se había considerado que las complicaciones neurológicas podían constituir una contraindicación al recambio valvular en fase aguda, ya que el riesgo de deterioro neurológico aumenta dramáticamente cuanto menos tiempo transcurre entre la manifestación neurológica y la intervención. No obstante, numerosas series defienden que la aparición de un evento neurológico es indicación "per se" para el tratamiento quirúrgico (14), mientras otros trabajos sugieren cierto beneficio con la cirugía en la fase aguda $(1,15)$ y otros sostienen que la embolia encefálica única no es indicación de cirugía cardiaca si la EI está controlada con tratamiento médico (3). En principio, ante la aparición de alteración neurológica asociada a la EI siempre se recomienda la realización de un estudio de imagen cerebral. Si se documenta la existencia de un infarto no hemorrágico se aconseja plantear cirugía esperando 2-4 semanas, si la situación lo permite; cuando se objetiva una hemorragia debe practicarse una angiografía cerebral para descartar la existencia de un aneurisma cerebral que requiera un abordaje neuroquirúrgico previo a la intervención cardiaca, que debería demorarse al menos 4-6 semanas; y si no se aprecian lesiones cerebrales (como en el caso de la meningitis asociada a la EI) se puede proceder a la sustitución valvular sin demora si existe indicación para la misma por existir mala evolución con tratamiento médico y/o factores de mal pronóstico en la fase activa (insuficiencia cardiaca refractaria; regurgitación valvular significativa; persistencia de sepsis; embolia sistémica recurrente; gérmenes virulentos de difícil erradicación con terapia médica; desarrollo de complicaciones mecánicas severas o fenómenos inmunológicos graves; prótesis valvular disfuncionante) (16-18).

En cualquier caso, el diagnóstico precoz y el rápido inicio de la terapia antibiótica constituyen el arma más efectivo para prevenir el desarrollo de complicaciones neurológicas y mejorar, por tanto, el pronóstico de los pacientes con EI.

\section{Bibliografía}

1. Heiro M, Nikoskelainen J, Engblom E, Kotilainen E, Marttila R, Kotilainen P. Neurologic manifestations of infective endocarditis: a 17-year experience in a teaching hospital in Finland. Arch Intern Med 2000; 160: 2781-7.

2. Kanter M, Hart R. Neurologic complications of infective endocarditis. Neurology 1991; 41: 1015-20.

3. Jones HR Jr, Siekert RG. Neurological manifestations of infective endocarditis. Review of clinical and therapeutic challanges. Brain 1989; 112: 1295-315.

4. Lerner PI. Neurologic complications of infective endocarditis. Med Clin North Am 1985; 69: 385-98.

5. Harrison MJ, Hampton JR. Neurological presentation of bacterial endocarditis. Br Med J 1967; 2: 148-51.

6. Thuny F, Di Salvo G, Belliard O, Avierinos JF, Pergola V, Rosenberg $\mathrm{V}$, et al. Risk of embolism and death in infective endocarditis: prognostic value of echocardiography: a prospective multicenter study. Circulation 2005; 112: 69-75.

7. Spelman D, Sexton DJ. Complications of infective endocarditis. UpToDate 14.22006.

8. Vilacosta I, Graupner C, San Roman JA, Sarria C, Ronderos R, Fernandez $\mathrm{C}$ et al. Risk of embolization after institution of antibiotic therapy for infective endocarditis. J Am Coll Cardiol 2002; 39: 1489-95.

9. Anderson DJ, Goldstein LB, Wilkinson WE, Corey GR, Cabell CH, Sanders LL et al. Stroke location, characterization, severity and outcome in mitral vs aortic valve endocarditis. Neurology 2003: 61: 1341-6.

10. Hart RG, Kagan-Hallet K, Joerns SE. Mechanism of intracranial hemorrhage in infective endocarditis. Stroke 1987; 18: 1048-56.

11. Aronin SI, Mukherjee SK, West JC, Cooney EL. Review of pneumococcal endocarditis in adults in the penicillin era. Clin Infect Dis 1998; 26: 165-71.

12. Lindberg JA. Endocarditis and normal cell meningitis caused by group B streptococci. Ugeskr Laeger 1998; 160: 6354-5.

13. Tornos P, Almirante B, Mirabet S, Permanyer G, Pahissa A, SolerSoler J. Infective endocarditis due to Staphylococcus aureus: deleterious effect of anticoagulant therapy. Arch Intern Med 1999; 159: 4735 .

14. Roder BL, Wandall DA, Espersen F, Frimodt-Moller N, Skinhoj P, Rosdahl VT. Neurologic manifestations in staphylococcus aureus endocarditis: a review of 260 bacteriemic cases in nondrug addicts. Am J Med 1997; 102: 379-86.

15. Parrino PE, Kron IL, Ross SD, Shockey KS, Kron AM, Towler MA, et al. Does a focal neurological deficit contraindicate operation in a patient with endocarditis? Ann Thorac Surg 1999; 67: 59-64.

16. Castillo Dominguez JC, Anguita Sanchez MP, Ramirez Moreno A, Siles Rubio JR, Torres Calvo F, Mesa Rubio D et al. The general characteristics and short- and long-term results of infective endocarditis in non-drug addicts. Rev Esp Cardiol 2000; 53: 344-52.

17. Horacio Casabe J, Deschle H, Cortes C, Stutzbach P, Hershson A, Nagel C et al. Predictors of hospital mortality in 186 cases of active infective endocarditis treated in a tertiary medical center (1992-2001). Rev Esp Cardiol 2003; 56: 543-5.

18. Angstwurm K, Borges AC, Halle E, Schielke E, Einhaupl KM, Weber JR. Timing the valve replacement in infective endocarditis involving the brain. J Neurol 2004; 251: 1220-6. 